\title{
Opioid prescribing after urologic surgery: we are part of the problem and part of the solution - an invited commentary on "an opioid prescription for men undergoing minor urologic surgery is associated with an increased risk of new persistent opioid use"
}

\author{
Matthew J. Ziegelmann ${ }^{1}$, Bridget L. Findlay ${ }^{1}$, Cameron J. Britton ${ }^{1}$, Elizabeth B. Habermann ${ }^{2}$ \\ ${ }^{1}$ Department of Urology, Mayo Clinic, Rochester, MN, USA; ${ }^{2}$ The Robert D. and Patricia E. Kern Center for the Science of Healthcare Delivery \\ Surgical Outcomes Program, Mayo Clinic, Rochester, MN, USA \\ Correspondence to: Matthew J. Ziegelmann. Department of Urology, Mayo Clinic, 200 1st Street SW, Rochester, MN 55905, USA. \\ Email: Ziegelmann.matthew@mayo.edu. \\ Provenance and Peer review: This article was commissioned by the editorial office, Translational Andrology and Urology. The article did not undergo \\ external peer review. \\ Comment on: Welk B, McClure JA, Clarke C, et al. An Opioid Prescription for Men Undergoing Minor Urologic Surgery Is Associated with an \\ Increased Risk of New Persistent Opioid Use. Eur Urol 2020;77:68-75.
}

Submitted Apr 17, 2020. Accepted for publication May 11, 2020.

doi: 10.21037/tau-2020-05

View this article at: http://dx.doi.org/10.21037/tau-2020-05

According to the United States (US) Center for Disease Control, an estimated 23,200 persons died in the US from prescription opioid overdose between 1999 and 2018 (1). In 2018 alone almost 15,000 prescription opioid-related deaths occurred, accounting for $32 \%$ of all drug overdose deaths (1). These concerns are not isolated to the US, as demonstrated by the United Nations' attempts to recognize and act upon global concerns related to drug misuse and abuse (2-4).

The opioid epidemic stems in large part from an overly optimistic outlook on addiction risks in patients with new exposure to opioid pain medications prescribed as part of routine medical care. A short letter published in the New England Fournal of Medicine in 1980 set off a dramatic change in the medical community's approach to pain management with unforeseen consequences (5). In just five sentences, the authors made the unsubstantiated conclusion that addiction is a rare phenomenon in patients who receive prescription narcotic medications without prior history of abuse. Countless authors would go on to cite this paragraph as evidence to support substantial increases in opioid prescribing that were realized over the subsequent three decades (6). As "pain" became known anecdotally as the "fifth vital sign", patient satisfaction became inextricably linked to this outcome (7). This led to an environment in which healthcare providers were incentivized to provide higher quantities and doses of opioids to ensure higher scores on patient satisfaction surveys, often linked to physician payment plans $(7,8)$. To add fuel to the fire, misguided motives driven by corporate profits further contributed to the drastic uptick in opioid prescribing (7).

There is ample evidence that surgeons have contributed to the current public health crisis through overprescribing in the postoperative period. Surgeons prescribe approximately $3-10 \%$ of all opioid medications (9-11). In some instances, this initial exposure can result in prolonged medication use, misuse, or abuse even after recovery from the incident procedure. Rates of persistent or prolonged opioid use in previously opioid-naïve surgical patients vary widely from $0.01 \%$ to $15 \%$ depending on cohort and outcome definitions $(12,13)$. Today more than ever, in the setting of astronomical health care expenditures, the potential downstream impact of opioid misuse is highly relevant. Lee $e t$ al. showed that new persistent opioid use postoperatively is associated with an average $\$ 1,500$ per patient increase in healthcare expenditures, resulting from more frequent hospital readmissions and ambulatory care visits (14). 
In the January 2020 issue of European Urology, Welk et al. evaluated the prevalence of persistent opioid use in a cohort of over 91,000 male patients who underwent one of several "minor" urologic surgeries (vasectomy, transurethral resection of the prostate, urethrotomy, hydrocelectomy, spermatocelectomy, and circumcision) between 2013-2016 in Ontario, Canada using multiple linkeddatasets (15). Analyses using these linked data sources are ripe for answering questions surrounding opioid prescribing patterns as there are mandatory reporting policies in place under the Canadian national healthcare system. To limit the impact of prior or chronic opioid exposure, patients with a history of opioid exposure within the preceding 180 days were excluded, along with those who were recently hospitalized or seen in the emergency department. This is similar to other definitions of "opioid-naïve" patients reported in the literature.

Within their cohort, $35 \%$ of the patients undergoing urologic procedures filled an opioid prescription within the first five days postoperatively. This is striking as the majority of these "minor" procedures are perceived to rarely if ever require opioids to manage pain, especially beyond the first few days after the procedure. The median morphine equivalents (MEQs) in those who received opioids was $113 \mathrm{mg}$, an amount that is significantly greater than reported and recommended for other minor/minimally invasive urologic procedures in modern-era guidelines $(16,17)$. This likely stems in part from the timeframe over which this analysis was derived. Surgeons' contribution to the opioid epidemic was only beginning to reach the forefront of public perception towards the end of the study period. In essence, the prescribing practices included in the current report represent findings in the era prior to our drive to promote opioid stewardship.

Persistent use in previously opioid-naïve patients, defined as filling at least two separate opioid prescriptions between 9-15 months after the incident urologic procedure, was seen in $1.6 \%$ of patients overall. Of those patients who filled an opioid prescription postoperatively, $2.02 \%$ had evidence of persistent use at $9-15$ months after the index procedure, compared with $1.35 \%$ of patients who did not immediately fill an opioid prescription [adjusted odds ratio 1.43 (95\% CI: 1.26-1.62, $\mathrm{P}<0.001)$. There was also a 3 -fold higher rate of emergency room visits and/or hospital admissions for opioid overdose in those patients who filled an opioid prescription postoperatively.

The rate of persistent opioid use identified by Welk et al. is notably lower than previous reports detailing outcomes in urologic surgery populations, where it has been estimated that $6-9 \%$ of patients show evidence of persistent use at three-months after surgery $(13,18,19)$. Using a similar dataset and rigorous methodology, Hosier and colleagues found a $9 \%$ rate of persistent opioid use at three-months postoperatively (after initial perioperative exposure) in a cohort of nearly 102,000 patients who underwent treatment for urolithiasis from 2013-2017 (20). In contrast, as Welk et al. emphasize, their definition of persistent is more restrictive compared to other studies, requiring at least two separate opioid medication prescription refills at $9-15$ months after surgery (15). The more restrictive definition likely accounts for some of the discrepancy seen in the rate of persistent use relative to other reports, but the conclusions drawn by Welk et al. remain extremely concerning. Specifically, receiving an opioid prescription within the immediate postoperative period, even after minor urologic procedures, was associated with a $43 \%$ increased odds of new persistent use beyond one year after surgery.

One could also surmise that opioid fills beyond 9-12 months postoperatively may be associated with an entirely unrelated circumstance. For example, a patient may undergo another procedure, or may develop an illness or injury resulting in the need for opioid medications. However, even at 3-6 months after surgery, Welk et al. identified a $33 \%$ increase in the odds of repeatedly filling opioid prescriptions in those patients who were exposed to opioids in the immediate postoperative period (15). To further assess the influence of other medical/surgical illness, the authors performed a sensitivity analysis to account for hospitalizations or other operative procedures occurring during the similar timeframe. This once again supported their outcomes [odds ratio 1.45 (95\% CI: 1.28-1.65; $\mathrm{P}<0.01)$ favoring a higher likelihood of persistent use in patients initially exposed to opioids postoperatively]. Additional models evaluated the impact of physician prescribing habits (i.e., those who were most likely to always or never prescribe opioids), medical comorbidities, and even a negative control assessment using the diagnoses of shingles or malignancy, lending further support to the rigor of their dataset and the reliability of the reported outcomes.

These findings are extremely important, and lend credence to our efforts to minimize opioid exposure for our patients whenever possible. Yet, there are limitations to the current analysis that must be acknowledged (15). For example, the quantity of opioids consumed by patients who 
filled their prescriptions is unknown. Previous work has shown that up to $60 \%$ of opioids prescribed at discharge from urologic surgery remained unused, suggesting a disconnect between patient needs and provider assumptions regarding postoperative pain (21). Further, non-opioid alternative interventions to minimize and manage pain control were not explored, despite their ability to contribute to pain management (15). Wide-spread clinician concerns regarding patient satisfaction and postoperative pain control are not unfounded, and urologists and other surgical specialists must balance optimizing pain control while minimizing risks of adverse medication-related outcomes for their patients. Patient satisfaction scores may be linked to certain measures of surgical quality, but, by the same token, opioid prescribing does not appear to be highlylinked with higher patient satisfaction scores in most instances (22).

Urologists are at the forefront in developing novel treatment protocols for postoperative pain. For example, multiple enhanced recovery after surgery (ERAS) protocols have been proposed to optimize perioperative and postoperative outcomes with the use of local anesthetic blocks, anti-inflammatories (ibuprofen, meloxicam, celecoxib), and anticonvulsants (gabapentin, pregabalin) $(23,24)$. Our group has also seen early success with implementation of an opioid guideline to promote standardization amongst larger groups of surgeons (25). These and other novel non-opioid pain regimens seem especially relevant for minor endo-urologic and penile/ scrotal cases where the degree and duration of anticipated postoperative pain is minimal, and they are just as likely to optimize patient satisfaction when compared to opioidbased regimens. Future work will enhance our ability to minimize untoward downstream effects while ensuring the best outcomes during the recovery process for our patients.

In conclusion, Welk et al. are to be congratulated for their important work assessing risks for persistent or prolonged opioid use in patients who undergo minor urological procedures. Their findings serve as an important reminder of the role urologists have in curbing the opioid epidemic. The challenge we face moving forward is toeing the line between appropriate postoperative pain management and the inherent risks with opioid medications as we seek to provide the highest level care for our patients.

\section{Acknowledgments}

Funding: None.

\section{Footnote}

Conflicts of Interest: All authors have completed the ICMJE uniform disclosure form (available at http://dx.doi. org/10.21037/tau-2020-05). MJZ reports personal fees from Paradigm Medical Communications, outside the submitted work. The other authors have no conflicts of interest to declare.

Ethical Statement: the authors are accountable for all aspects of the work in ensuring that questions related to the accuracy or integrity of any part of the work are appropriately investigated and resolved.

Open Access Statement: This is an Open Access article distributed in accordance with the Creative Commons Attribution-NonCommercial-NoDerivs 4.0 International License (CC BY-NC-ND 4.0), which permits the noncommercial replication and distribution of the article with the strict proviso that no changes or edits are made and the original work is properly cited (including links to both the formal publication through the relevant DOI and the license). See: https://creativecommons.org/licenses/by-nc-nd/4.0/.

\section{References}

1. Control CfD, Prevention. a. 2019 Annual Surveillance Report of Drug-Related Risks and Outcomes - United States Surveillance Special Report. Centers for Disease Control and Prevention, U.S. Department of Health and Human Services.

2. Becker WC, Fiellin DA. Limited evidence, faulty reasoning, and potential for a global opioid crisis. BMJ 2017;358:j3115.

3. Berterame S, Erthal J, Thomas J, et al. Use of and barriers to access to opioid analgesics: a worldwide, regional, and national study. Lancet 2016;387:1644-56.

4. Crime UNOoDa. Outcome Document of the 2016 United Nations General Assembly Special Session on the World Drug Problem: Our Joint Commitment to Effectively Addressing and Countering the World Drug Problem (April 19 - 21, 2016).

5. Porter J, Jick H. Addiction rare in patients treated with narcotics. N Engl J Med 1980;302:123.

6. Leung PTM, Macdonald EM, Stanbrook MB, et al. A 1980 Letter on the Risk of Opioid Addiction. N Engl J Med 2017;376:2194-5.

7. Jones GH, Bruera E, Abdi S, et al. The opioid epidemic 
in the United States-Overview, origins, and potential solutions. Cancer 2018;124:4279-86.

8. Afilalo $M$, Tselios C. Pain relief versus patient satisfaction. Ann Emerg Med 1996;27:436-8.

9. Levy B, Paulozzi L, Mack KA, et al. Trends in Opioid Analgesic-Prescribing Rates by Specialty, U.S., 2007-2012. Am J Prev Med 2015;49:409-13.

10. Guy GP Jr, Zhang K. Opioid Prescribing by Specialty and Volume in the U.S. Am J Prev Med 2018;55:e153-5.

11. Weiner SG, Baker O, Rodgers AF, et al. Opioid Prescriptions by Specialty in Ohio, 2010-2014. Pain Med 2018;19:978-89.

12. Jivraj NK, Raghavji F, Bethell J, et al. Persistent Postoperative Opioid Use: A Systematic Literature Search of Definitions and Population-based Cohort Study. Anesthesiology 2020;132:1528-39.

13. Shkolyar E, Shih IF, Li Y, et al. Robot-Assisted Radical Prostatectomy Associated with Decreased Persistent Postoperative Opioid Use. J Endourol. 2020;34:475-81.

14. Lee JS, Vu JV, Edelman AL, et al. Health Care Spending and New Persistent Opioid Use After Surgery. Ann Surg 2019. [Epub ahead of print].

15. Welk B, McClure JA, Clarke C, et al. An Opioid Prescription for Men Undergoing Minor Urologic Surgery Is Associated with an Increased Risk of New Persistent Opioid Use. Eur Urol 2020;77:68-75.

16. Koo K, Faisal F, Gupta N, et al. Recommendations for Opioid Prescribing after Endourological and Minimally Invasive Urological Surgery: An Expert Panel Consensus. J Urol 2020;203:151-8.

17. Overton HN, Hanna MN, Bruhn WE, et al. OpioidPrescribing Guidelines for Common Surgical

Cite this article as: Ziegelmann MJ, Findlay BL, Britton CJ, Habermann EB. Opioid prescribing after urologic surgery: we are part of the problem and part of the solution—an invited commentary on "an opioid prescription for men undergoing minor urologic surgery is associated with an increased risk of new persistent opioid use". Transl Androl Urol 2020;9(3):9971000. doi:10.21037/tau-2020-05
Procedures: An Expert Panel Consensus. J Am Coll Surg 2018;227:411-8.

18. Tam CA, Dauw CA, Ghani KR, et al. New Persistent Opioid Use After Outpatient Ureteroscopy for Upper Tract Stone Treatment. Urology 2019;134:103-8.

19. Barham DW, McMann LP, Musser JE, et al. Routine Prescription of Opioids for Post-Vasectomy Pain Control Associated with Persistent Use. J Urol 2019;202:806-10.

20. Hosier GW, McGregor T, Beiko D, et al. Persistent Opioid Use Among Patients with Urolithiasis: A Population based Study. Eur Urol Focus 2020;6:745-51.

21. Theisen KM, Myrga JM, Hale N, et al. Excessive Opioid Prescribing After Major Urologic Procedures. Urology 2019;123:101-7.

22. North F, Crane SJ, Ebbert JO, et al. Do primary care providers who prescribe more opioids have higher patient panel satisfaction scores? SAGE Open Med 2018;6:2050312118782547.

23. Lucas J, Gross M, Yafi F, et al. A Multi-institutional Assessment of Multimodal Analgesia in Penile Implant Recipients Demonstrates Dramatic Reduction in Pain Scores and Narcotic Usage. J Sex Med 2020;17:518-25.

24. Mehta A, Hsiao W, King P, et al. Perioperative celecoxib decreases opioid use in patients undergoing testicular surgery: a randomized, double-blind, placebo controlled trial. J Urol 2013;190:1834-8.

25. Ziegelmann M, Joseph J, Glasgow A, et al. Comparison of prescribing patterns before and after implementation of evidence-based opioid prescribing guidelines for the postoperative urologic surgery patient. Am J Surg 2019. doi: 10.1016/j.amjsurg.2019.11.037. 